\title{
Influence des larves séchées de mouches domestiques (Musca domestica, L.) sur la prise alimentaire du poulet local (Gallus domesticus, L.) au Burkina Faso.
}

TRAORÉ Ibrahima ${ }^{1 *}$, POUSGA Salimata ${ }^{1}$, SANKARA Fernand ${ }^{1}$, ZONGO Zoram Gouda ${ }^{1}$, COULIBALY Kalifa ${ }^{1}$, NACOULMA Jacques-Philippe ${ }^{1}$, KENIS Marc ${ }^{2}$, OUÉDRAOGO Georges Anicet ${ }^{1}$.

1 Institut du Développement Rural (IDR), Université Nazi Boni (UNB), BP : 1091, Bobo Dioulasso, Burkina Faso ${ }^{\circ}$;

${ }^{2}$ Centre for Agricultural Bioscience International (CABI), Rue des Grillons 1, 2800 Delémont, Switzerland;

*Auteur pour la correspondance : Tél. : +226 70360069 ; Email :phenix078@gmail.com.

Mots-clés : Aviculture traditionnelle, asticots, alimentation au choix, cafeteria, Burkina Faso. Keywords $^{\circ}$ : Traditional poultry farming, maggots, choice-feeding, cafeteria, Burkina Faso.

1 RÉSUMÉ

Cette étude a été réalisée afin d'appréhender les préférences alimentaires des poulets locaux nourris au choix en présence des asticots séchés, dans le but de promouvoir l'utilisation des larves de la mouche domestique (Musca domestica, L.) comme source de protéine animale dans l'alimentation de la volaille locale. Pour ce faire, un essai a été réalisé durant 6 semaines avec 54 poulets locaux âgés de 18 mois. Ces oiseaux ont été pesés et répartis de façon aléatoire en trois traitements représentant les périodes de la journée pendant lesquelles un test de cafeteria a été conduit $(08 \mathrm{~h}, 12 \mathrm{~h}$ et $15 \mathrm{~h})$. Les aliments étaient constitués d'asticots séchés, de grains de maïs concassés et d'un aliment complet ponte, servis dans des mangeoires différentes au même moment. Chaque test de cafeteria durait 30 mn au cours desquelles une observation directe et un film étaient effectués. Les consommations alimentaires individuelles, la proportion de chaque type d'aliment dans la ration et les caractéristiques des œufs ont été déterminés. Les résultats ont montré des consommations moyennes d'asticots séchés de 5,23 $\pm 2,59 \mathrm{~g} \mathrm{MS} /$ individu/jour pour les traitements de $12 \mathrm{~h}$, de 4,68 \pm 1,81 $\mathrm{g} \mathrm{MS} /$ individu/jour pour les groupes de $08 \mathrm{~h}$ et de 4,60 \pm $1,67 \mathrm{~g} \mathrm{MS} /$ individu/jour pour le traitement de $15 \mathrm{~h}$. Concernant les caractéristiques des œufs, les valeurs numériques du poids moyen, du jaune d'œuf ainsi que la coloration du jaune ont été légèrement supérieures au niveau du traitement de $12 \mathrm{~h}$ comparativement aux autres. Cette étude a permis de montrer que les asticots sont appréciés par les poulets et beaucoup plus à midi. De tels résultats, mis à la disposition des aviculteurs, leurs permettront de cibler la période propice pour une complémentation efficiente en protéines et de réduire les charges liées à l'acquisition des intrants protéiques inaccessibles en milieu traditionnel. 
Influence of dried fly larvae (Musca domestica, L.) on the food intake of local chicken (Gallus domesticus, L.) in Burkina Faso.

\begin{abstract}
This study was carried out to investigate the feeding behaviour of local chickens choice-fed with different feed including dried maggots. The objective is to promote the use of Musca domestica larvae as animal source protein in traditional poultry feeding in Burkina Faso. Feeding experiments were done with 54 local chickens aged 18 months old, over six weeks. The birds were weighed and randomly divided into three treatment groups representing the time of the day the cafeteria trial was conducted. These times were $08 \mathrm{am}, 12$ noon and 03 $\mathrm{pm}$. The choices offered in the cafeteria trial were dried fly larvae, cracked maize grains and a balanced layers' diet, given in separated feeders. Each cafeteria test lasted 30 minutes during which direct observation and filming were done. The total individual feed intake, the proportion of each cafeteria feed to the total individual feed intake and egg characteristics were determined. The average dried maggot's consumption at 12 noon $(5.23 \pm 2.59 \mathrm{~g}$ $\mathrm{DM}$ /individual/day) was significantly higher than the amount consumed at 08 am (4.68 \pm $1.81 \mathrm{~g} \mathrm{DM} /$ individual/day) and $03 \mathrm{pm}(4.60 \pm 1.67 \mathrm{~g} \mathrm{DM} /$ individual/day $)(P<0.05)$. No significant difference was seen between treatments concerning egg parameters. However, the numerical values for average egg weight, yolk weight and yolk color were slightly higher in the group tested at 12 noon compared to the other treatments. The study shows that maggots are preferred by the chickens, and seem to be well preferred at noon. These results will allow poultry farmers to target the right time for efficient supplementation using dried fly larvae and contributing to reduce the cost linked to the acquisition of protein inputs that are traditionally inaccessible.
\end{abstract}

\section{INTRODUCTION}

En Afrique subsaharienne, le développement de l'aviculture est entravé par plusieurs contraintes dont la principale est l'accès aux aliments sources de protéine (Ouattara et al., 2016; Pousga et al., 2019). Au Burkina Faso, l'aviculture traditionnelle qui fournit plus de 98\% de l'effectif avicole (MRA, 2015) souffre considérablement de la pénurie d'approvisionnement en ces intrants. En effet, les ingrédients à fort taux de protéines (la farine de poisson, les tourteaux de coton, de soja et d'arachide) disponibles sont pour la plupart des produits d'exportations et inaccessibles notamment en milieu rural (Pousga et al., 2007 ; Ouattara et al., 2016). Par conséquent, la volaille locale peu performante est élevée dans des conditions d'alimentation précaires (Ouattara et al., 2016 ; Pousga et al., 2019). Les termites sont fréquemment utilisés comme apport protéinique en aviculture traditionnelle, mais leur disponibilité est aléatoire et les quantités disponibles sont souvent insuffisantes (Kenis et al., 2014 ; Sankara et al., 2018 ; Dao et al., 2020). L'identification de nouvelles sources de protéines animales à moindre coût s'avère une nécessité pour endiguer cette préoccupation dont l'impact sur les productions avicoles n'est pas à négliger. C'est ainsi que les larves de mouche domestique (Musca domestica, L.) sont apparues ces dernières années comme une alternative aux protéines importées (Van Huis et al., 2013; Kenis et al., 2018). Ces insectes offrent l'avantage d'être présents presque dans tous les écosystèmes, et leurs larves sont également ingérées par la volaille divagante, ce qui témoigne de leur grande importance pour l'aviculture (Van Huis et al., 2013). Plusieurs travaux ont aussi montré leur capacité à remplacer efficacement les sources conventionnelles de protéine, la farine de poisson en particulier, dans l'alimentation de la volaille sans diminuer leurs performances (Van 
Huis et al., 2013 ; Kenis et al., 2014, 2018 ; Pousga et al., 2019). Les asticots sont susceptibles de se développer sur beaucoup de substrats qui n'ont pas un grand intérêt pour l'homme (Pomalegni et al., 2016 ; Sanou et al., 2019b). Mieux, ils entrainent après leur passage la dégradation poussée du substrat de production fournissant ainsi des résidus organiques de qualité appréciable pour la fertilisation du sol (Bloukounon-Goubalan et al., 2019). Par ailleurs, Pomalégni et al. (2016) au Benin et Sanou et al. (2019b) au Burkina Faso ont montré qu'environ respectivement $87,41 \%$ et $61,4 \%$ des producteurs enquêtés avaient une bonne perception des asticots et étaient favorables à leur utilisation dans l'alimentation de la volaille locale. La présente étude vise à promouvoir l'utilisation des larves de mouche domestique en aviculture traditionnelle. Cette

\section{MATERIEL ET METHODES}

3.1 Présentation du site d'étude: La présente étude a été menée dans l'animalerie de l'Université Nazi Boni située dans le village de Nasso $\left(11^{\circ} 12^{\prime} \mathrm{N}, 4^{\circ} 26^{\prime} \mathrm{O}\right)$. Ce village relève de la commune de Bobo-Dioulasso dans la région des Hauts Bassins où le climat est de type sudsoudanien et caractérisé par une saison sèche (octobre à avril) et une saison pluvieuse (Mai à Septembre). La saison sèche est marquée par une période froide (novembre à janvier) et une période chaude (février à avril). Les pluies sont inégalement réparties dans le temps et dans l'espace et relativement abondantes (environ $748 \mathrm{~mm}$ d'eau en 82 jours de pluie en 2017) (INSD, 2018). La végétation est constituée de savanes boisées, arborées et arbustives (Guinko, 1984). Les sols sont très favorables à l'agriculture et le relief, peu accidenté, se caractérise par une chaîne rocheuse au sud, des bas-fonds et des plaines aménageables. Le réseau hydrographique est riche d'une vingtaine de sources.

3.2 Le poulailler et ses équipements : Le poulailler est un bâtiment de $10 \mathrm{~m}$ sur $10 \mathrm{~m}$ subdivisé en trois lots aménagés chacun en quatre box, soit un total de 12 box. Les box ont étude complète celle réalisée récemment par Traoré et al. (2020) chez la pintade locale où la préférence pour les asticots séchés servis concurrentiellement avec d'autres aliments (le sorgho, les grains de maïs concassés et un aliment complet ponte) a également été évaluée. Les résultats ont montré un faible niveau d'ingestion des asticots séchés (moins de 5\%) comparativement aux céréales. Il s'agira spécifiquement (i) d'apprécier le comportement alimentaire des poulets locaux dans un système d'alimentation au choix (mode cafeteria) avec différents types d'aliments (asticots séchés, grains de maïs concassé, aliment complet ponte), (ii) d'estimer le moment idéal de la journée pour faire une complémentation en asticots et (iii) d'évaluer l'effet de leur consommation sur les caractéristiques des œufs de la poule locale en ponte.

été faits de grillage. La toiture faite en tôle a une hauteur au centre de $5 \mathrm{~m}$. La ventilation du bâtiment était naturelle et son sol couvert d'une litière constituée de glumelles de riz. Dans chaque box, étaient disposés un abreuvoir de capacité six litres et trois mangeoires identiques lors $\mathrm{du}$ test de nutrition. L'extérieur du bâtiment était clôturé par du grillage offrant ainsi une aire de récréation pour la volaille.

\subsection{Les animaux expérimentaux :} L'expérimentation a été réalisée sur 54 poulets locaux d'environ 18 mois d'âge, dont 48 poules en ponte et six coqs. Ces oiseaux ont été acquis auprès d'un éleveur de Siniéna, un village situé dans la commune de Banfora (Province de la Comoé, Région des Cascades) à l'Ouest du Burkina Faso. Ils y ont été élevés en semiclaustration et nourris avec du maïs concassé complémenté occasionnellement de termites. Ces poulets ont également bénéficiés d'une prophylaxie complète. Des larves de mouche domestique $(M$. domestica) ont été utilisées sous leur forme séchée.

3.4 Production des asticots : Les asticots ont été produits selon les indications rapportées par Sanou et al. (2019a). Un mélange de quatre 
$\mathrm{Kg}$ de litière sale avec sept litres d'eau a été réalisé dans un bac métallique servant de récipient de production. Après $24 \mathrm{~h}$ d'exposition à la ponte naturelle de mouches, ce mélange a été couvert à l'aide d'une bâche faite en sac de céréales offrant un milieu aéroanaérobique. Les asticots ont été récoltés le 5ème jour après exposition à l'aide de tamis de maille adaptée, en exploitant le caractère lucifuge des asticots. Ils ont été nettoyés et séchés au soleil dans les bacs pendant au moins deux jours en fonction de l'intensité du soleil.

\subsection{Les aliments expérimentaux utilisés}

Trois types d'aliments ont été utilisés pour ce test :

- $\quad$ les asticots séchés : ce sont des larves de M. domestica récoltées et séchées au soleil. Elles ont été servies entières sous cette forme séchée aux poulets ;

- le maïs : le maïs jaune a été concassé afin de rendre la préhension facile par les poulets ;

un aliment complet: il s'agit d'une ration ponte formulée à partir du logiciel UNEForm Monogastriques avec différents ingrédients dont la source de protéine animale a été la farine de poisson. Il a constitué la ration de base servie durant toute la période du test.

Ces aliments ont été servis aux poulets lors du test de nutrition dans les mêmes proportions de $50 \mathrm{~g} / \mathrm{box} /$ jour dans des mangeoires différentes. Le Tableau 1 présente la formule et la valeur nutritionnelle de l'aliment complet ponte ayant servi lors des tests et comme ration de base. L'ensemble des ingrédients utilisés a été acheté auprès des revendeurs ou d'un provendier sur la place du marché dans la ville de Bobo Dioulasso.

3.6 Dispositif expérimental : Les oiseaux ont été répartis au hasard en trois traitements de 18 sujets chacun. Chaque traitement comportait quatre répétitions (correspondant aux 12 box) dont deux répétitions comprenant quatre poules et un coq et les deux autres, trois poules et un coq. Durant l'essai, l'eau était distribuée ad libitum (à volonté). Les aliments ont été servis dans les mangeoires et disposés dans le même ordre (maïs concassé-aliment complet ponte-asticots séchés) pendant toute la durée de l'expérience (Fig. 1). Le comportement de la volaille a également été enregistré durant le test par filmage. Ces films ont été faits dès que les poulets ont eu accès aux trois aliments; l'opérateur se plaçant dans un angle du box.

3.7 Influence de la période de service des aliments sur la prise alimentaire du poulet: La détermination du moment idéal de la journée pour une complémentation en asticots séchés a été réalisée selon le mode cafeteria. Le principe est de servir, dans le même temps, plusieurs aliments dans un seul récipient comportant un certain nombre de plages pour aliments à l'image de l'assiette anglaise ou dans autant de récipients disposés côte à côte. Le test a duré 6 semaines (du 22 Janvier au 4 Mars 2017) et les traitements ont été les moments de la journée ( 08 h, 12 h et 15 h) auxquels les oiseaux avaient accès aux trois aliments (asticots séchés, grains de maïs concassé et aliment complet ponte). Ces aliments ont été servis dans les mêmes proportions de $50 \mathrm{~g} /$ box/jour. La durée d'accès était de 30 minutes au bout desquelles les éventuels restes d'aliments étaient retirés. Seul l'aliment complet a été distribué ad libitum durant le reste de la journée (pour la cafeteria de $08 \mathrm{~h}$ ), le matin jusqu'à l'heure du test et après (les cafeterias de $12 \mathrm{~h}$ et $15 \mathrm{~h}$ ).

La quantité d'aliment de la ration de base destinée à chaque box et servie hors de la cafeteria a été répartie en trois, soit la même fréquence de distribution par jour (matin, midi et soir). Toutefois, chaque sujet a eu droit à 70 $\mathrm{g}$ de l'aliment complet par jour. Les restes d'aliment ont été relevés et pesés après chaque cafeteria et quotidiennement tous les matins avant la distribution de la ration journalière. Pour l'étude des caractéristiques, quatre œufs par traitement ont été récoltés par semaine (soit un total de 12 œufs/semaine). Un séparateur de jaune d'œuf a été utilisé pour dissocier le blanc du jaune d'œuf. L'éventail colorimétrique (DSM Yolk Color Fan ${ }^{\mathrm{TM}}$ ), muni d'une échelle de 
couleur graduée de 1 à 15 , a été utilisé pour apprécier la couleur du jaune d'œuf.

3.8 Collecte des données : Pendant toute la durée du test, les paramètres suivants ont été calculés grâce aux différentes formules :

les quantités d'aliment consommées au niveau de chaque lot:

$\mathrm{Qc}=[(\mathrm{Q} s \times \mathrm{Ks})-(\mathrm{Qr} \times \mathrm{Kr})]$

où : $Q \mathrm{c}=$ quantité d'aliment consommé ( $\mathrm{g}$ MS), Qs = quantité servie (g), Qr = quantité refusé ( $\mathrm{g})$, Ks et $\mathrm{Kr}$ les taux de matière sèche (MS) des aliments servi et refusé respectivement.

- la proportion de chaque aliment consommé :

Taux (\%) de l'aliment X = (Qc X / Qc T) x 100 où : $\mathrm{Qc} X=$ quantité de l'aliment $\mathrm{X}$ consommé (g MS) et Qc T = quantité totale consommée pendant la cafeteria (g MS).

- L'énergie métabolisable (EM) des différentes ingestions alimentaires (INRA, 1987) :

$\mathrm{EM}(\mathrm{Kcal} / \mathrm{kg}$ de MS $)=3951+54,4 \mathrm{MG}-88,7$ CB - 40,8 MM

où : $\mathrm{MG}=$ matière grasse, $\mathrm{CB}=$ cellulose brute, $\mathrm{MM}=$ matières minérales.

Des prélèvements de chaque ration (100 g) ont été effectués et envoyés au laboratoire de nutrition animale de l'INERA (Institut National de l'Environnement et des Recherches

\section{RESULTATS}

4.1 Étude de la composition chimique des aliments utilisés : La teneur en protéine brute (PB) décroît des asticots séchés aux grains de maïs concassé (Tableau 2). Les asticots et l'aliment complet ont un taux de matières minérales plus élevé que le maïs concassé. En revanche, ce dernier aliment est plus énergétique que les deux autres. Les asticots séchés présentent plus de matières grasses (MG) et les teneurs les plus faibles de phosphore $(\mathrm{P})$ et de calcium $(\mathrm{Ca})$.

4.2 Influence de la période de service sur la prise alimentaire journalière

4.2.1 Variation des quantités d'aliments consommés en fonction de la période de
Agricoles) de Kamboincé à Ouagadougou pour la détermination de la composition chimique des rations. Les teneurs en matière sèche (MS), en matière organique (MO), en matières grasses (MG), en protéines brutes (PB) et en matières minérales $(\mathrm{MM})$ ainsi que le taux de cellulose brute (CB) y ont été déterminées selon les méthodes standards (AOAC, 1995).

- $\quad$ Les poids des œufs, de la coquille, du jaune et du blanc d'œuf ont été mesurés ;

- La coloration du jaune d'œuf a été appréciée grâce à l'éventail colorimétrique (DSM Roch Color Fan ${ }^{\mathrm{TM}}$ ) sur une échelle de 1 à 15 .

3.9 Analyses statistiques: Les analyses statistiques ont été faites par les logiciels $\mathrm{R}$ version 3.6.2 et Microsoft Excel version 2013. Le logiciel $\mathrm{R}$ version 3.6.2 a été utilisé pour la statistique descriptive, l'analyse de variance (ANOVA) et les comparaisons des moyennes. Le test de Student au seuil de signification de $5 \%$ a servi pour la comparaison des données quantitatives à savoir les consommations alimentaires et les poids des œufs, de la coquille, $\mathrm{du}$ blanc et du jaune d'œuf. Pour les comparaisons des taux de consommations et des moyennes de l'indice de coloration (donnée qualitative), le test de Khi deux au seuil de $5 \%$ a été réalisé. Les graphiques ont été générés grâce au tableur Excel version 2013.

service: Les quantités moyennes d'aliments consommés par individu et par jour durant les cafeterias sont présentées dans le Tableau 3. La quantité moyenne globale d'asticots consommée a été de 4,84 g MS. Celles du maïs concassé et de l'aliment complet s'élèvent respectivement à 6,06 g MS et 5,97 g MS. La consommation moyenne globale par jour et par individu des cafeterias a été de 16,87 g MS. L'analyse de variance montre qu'il y a une différence significative $(p<0,05)$ entre les traitements du test alimentaire. Le traitement de $12 \mathrm{~h}$ présente la quantité d'asticots séchés ingérés la plus élevée $(5,23 \mathrm{~g}$ MS/individu/jour), suivi respectivement des 
traitements de $08 \mathrm{~h}$ (4,68 g MS/individu/jour) et $15 \mathrm{~h}$ (4,60 g MS/individu/jour) dont les niveaux d'ingestions sont comparables ( $\mathrm{p}>$ $0,05)$. En ce qui concerne le maïs concassé, les ingestions moyennes ont été de 4,83; 7,19 et $6,16 \mathrm{~g} \mathrm{MS} /$ individu/jour respectivement pour les traitements de $08 \mathrm{~h}, 12 \mathrm{~h}$ et $15 \mathrm{~h}$. Tous les traitements ont été statistiquement $(p<0,05)$ différents. Pour la consommation en aliment complet durant la cafeteria, les traitements de

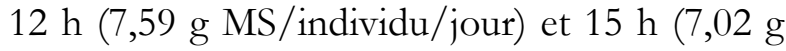
MS/individu/jour) ont été sensiblement égales ( $p>0,05)$. Par ailleurs, ils ont présenté des valeurs significativement supérieures $(\mathrm{p}<0,05)$ à celle du traitement de $8 \mathrm{~h} \quad(3,32 \mathrm{~g}$ MS/individu/jour). De façon générale, le maïs concassé $(35,92 \%)$ et l'aliment complet $(35,39 \%)$ ont été les plus consommés que les asticots séchés $(28,69 \%)$ durant les tests alimentaires (Tableau 4). La Figure 2 présente l'évolution des quantités d'aliment consommé pendant la cafeteria et pour chaque traitement durant les six semaines.

\subsubsection{Variation de l'ingestion de l'aliment} complet servi après les cafeterias: Le Tableau 5 montre les quantités d'aliment complet consommées après chaque cafeteria. L'ingestion moyenne par jour et par individu a été de 47,05 g MS. Il n'y a pas eu de différence significative $(p>0,05)$ entre les traitements de $12 \mathrm{~h}(49,25 \mathrm{~g}$ MS/individu/jour) et de $15 \mathrm{~h}$ (48,14 g MS/individu/jour). Cependant, ceux-ci étaient statistiquement différents et supérieurs $(\mathrm{p}<0,05)$ au traitement de $08 \mathrm{~h}(43,77 \mathrm{~g}$ MS/individu/jour).

4.2.3 Variation de la quantité journalière totale d'aliment consommé selon le traitement : La consommation alimentaire journalière totale a été en moyenne de $63,93 \mathrm{~g}$ MS dont 26,39\% pour la cafeteria (Tableau 5). Elle n'a pas significativement varié $(p>0,05)$

\section{DISCUSSION}

5.1 Étude de la variation de la composition chimique des aliments utilisés : Les résultats d'analyse des aliments utilisés dans cette étude montrent que le maïs entre les traitements de $12 \mathrm{~h} \quad(69,26 \mathrm{~g}$ MS/individu/jour) et de $15 \mathrm{~h} \quad(65,92 \mathrm{~g}$ $\mathrm{MS} /$ individu/jour). Néanmoins, ces niveaux d'ingestion ont été supérieurs et statistiquement différents $(p<0,05)$ de celui du traitement de $08 \mathrm{~h}$ (56,60 g MS/individu/jour).

4.2.4 Estimation des quantités de nutriments fournis par les différentes rations: Le Tableau 6 donne les quantités de nutriments apportés par les aliments ingérés lors des différents traitements. Le traitement de $12 \mathrm{~h}$ présente les valeurs les plus élevées sauf pour l'énergie métabolisable.

4.3 Étude de la répartition spatiotemporelle des oiseaux en fonction des préférences alimentaires : L'observation directe et l'analyse des films ont permis de distinguer trois phases dans le comportement des poulets autour des mangeoires. Du début du test jusqu'à la 4ème semaine, les poulets étaient attirés en premier par les mangeoires où se trouvait le maïs concassé. Puis, entre la 4ème et la 5ème semaine, leur distribution a été plus ou moins homogène autour des trois mangeoires. De la 5ème semaine à la fin du test, tous les poulets se dirigeaient premièrement vers les mangeoires contenant les larves. Cette répartition temporelle se répercute sur les consommations globales enregistrées à travers la fluctuation des quantités ingérées.

4.4 Effet des traitements sur les caractéristiques des œufs: Le Tableau 7 présente la statistique des paramètres des œufs étudiés durant le test. Le moment de la complémentation au choix n'a pas significativement $(\mathrm{p}>0,05)$ influencé la qualité des œufs bien que les valeurs des différentes caractéristiques aient été légèrement supérieures au niveau du traitement de $12 \mathrm{~h}$ comparativement aux autres ( $08 \mathrm{~h}$ et $15 \mathrm{~h}$ ).

concassé, les asticots séchés et l'aliment complet peuvent être respectivement considérés comme des sources d'énergie, de protéines et de minéraux. Le taux de protéines 
brutes des asticots séchés de cette étude est proche des 50,4\% obtenu par Makkar et al. (2014) à l'issu d'une synthèse de 29 études sur la mouche domestique. Toutefois, la teneur en lipides ainsi que l'énergie métabolisable des asticots séchés sont inférieures à celles rapportées par les mêmes auteurs qui ont enregistré des valeurs de $18,9 \%$ et 5469,57 $\mathrm{Kcal} / \mathrm{kg}$ MS respectivement. Ces différences pourraient être associées au support nutritif de production utilisé, à la durée de stockage après séchage et au mode de séchage (Fasakin et al., 2003 ; Tendonkeng et al., 2017).

5.2 Influence de la période de service sur la consommation des différents types d'aliments : Les consommations moyennes globales des aliments au cours du test ont enregistré des différences significatives ( $p<$ 0,05). La quantité d'asticots séchés ingérée a été la plus faible sauf au niveau du traitement de 08 $h$ où les niveaux d'ingestion sont similaires. Les différences de consommation observées peuvent s'expliquer par l'habitude alimentaire, l'aspect de l'aliment (granulés ou farine), l'état et les besoins physiologiques ainsi que les préférences alimentaires. Ces facteurs sont susceptibles d'influencer le niveau d'ingestion des aliments par la volaille (Picard et al., 2000 ; Bouvarel et al., 2010). En effet, les poulets étant des granivores et aussi élevés en milieu traditionnel, les céréales constituent leur aliment de base. Par conséquent, ils ont l'habitude du maïs concassé par rapport aux asticots séchés et à l'aliment complet dont les caractéristiques diffèrent de celles du maïs utilisé. L'aspect granulé du maïs explique sa forte consommation avec des valeurs souvent supérieures. Des études antérieures avaient montré que les aliments farineux étaient difficilement préhensibles par la volaille et qu'ils étaient moins consommés que ceux granuleux (Picard et al., 2000 ; Bouvarel et al., 2010). L'état physiologique des poulets (poules en ponte) en plus de la nécessité de satisfaire les besoins énergétiques ont pu influer sur les niveaux de consommation occasionnant de fortes variations individuelles au sein d'un même traitement et entre les traitements. En effet, avec l'instinct de couvaison de certaines poules chez lesquelles la ponte s'est arrêtée, leurs prises alimentaires ont considérablement baissé notamment pour la source de protéine. Cela a été perceptible dans leur préférence pour le maïs concassé (aliment le plus énergétique) dans la même période. La satisfaction des équilibres énergétique et minéral est alors devenue l'option préférentielle chez ces poules (Bouvarel et al., 2010, 2014). Les poulets ont beaucoup plus ingéré les asticots séchés en quantité plus ou moins égale aux deux aliments classiques (aliment complet et le maïs concassé) au niveau du traitement de $12 \mathrm{~h}$ que dans les traitements de $08 \mathrm{~h}$ et $15 \mathrm{~h}$. Ce qui pourrait s'expliquer par la très grande variabilité des besoins alimentaires en rapport avec les périodes de services dans la journée. Toutefois, l'ingestion des asticots séchés a été faible comparativement aux deux autres aliments. Cela serait dû au mode d'alimentation utilisé car les oiseaux, de par leur nature granivore et avec l'habitude alimentaire, seront attirés par les céréales dans une situation de choix. Contrairement à la présente étude, Traoré et al. (2020) ont relevé chez la pintade locale des ingestions d'asticots séchés plus de six fois plus faible dans des conditions d'expérimentation similaires. Ces auteurs ont rapporté une ingestion moyenne d'asticots séchés de $0,71 \mathrm{~g}$ MS sur l'ensemble de leur étude. Ce qui montre que les asticots séchés sont une denrée appréciée des poulets, contrairement aux pintades, qui peut être donnée en complément du maïs. En rapport avec les courbes d'évolution des différents aliments ingérés, les tendances pour le traitement de 08 h (Fig. 2 A) montrent que la consommation des asticots séchés est intermédiaire à celles du maïs concassé et de l'aliment complet jusqu'à la moitié de la 4ème semaine. Au-delà de cette date, la quantité d'asticots ingérée devient supérieure à celle des deux autres aliments. Par contre, pour les traitements de $12 \mathrm{~h}$ (Fig. 2 B) et 15 h (Fig. 2 C), la consommation d'asticots est légèrement inférieure à celle des autres aliments. La 
quantité de maïs concassé consommée subit une hausse à partir de la 5ème semaine aussi bien pour ces deux derniers traitements que celui de 08 h. L'analyse de ces courbes de consommation, montre qu'elles semblent se rapprocher au-delà de la 5ème semaine pour tous les traitements. Cette convergence des courbes de consommation indiquerait le fait qu'en libre choix, les oiseaux à travers leur instinct sélectionnent les aliments au vu de créer un équilibre répondant à leur besoin (Fanatico et al., 2013, 2016). Ainsi, chaque type d'aliment apporte sa part contributive dans l'ingestion globale de sorte à assurer l'équilibre énergétique, l'homéostasie protéique et l'homéothermie (Bouvarel et al., 2010). Pour ces auteurs, la consommation à long terme aboutit à une régulation d'aliment à aliment, dépendant des processus métaboliques et visant en premier lieu à contrebalancer les dépenses énergétiques. Le même point de vue avait été soutenu par Pousga et al. (2005) lors d'une étude similaire à la nôtre sur l'alimentation de la volaille en mode cafeteria. La consommation alimentaire journalière moyenne enregistrée dans cette étude est comparable à celles rapportées par Pousga et al. (2019). Ces auteurs ont enregistré des consommations alimentaires journalières variant de 65,10 $\pm 2,01$ à $66,80 \pm 3,05 \mathrm{~g}$ MS chez des poulets en croissance dont la ration a été complémentée par deux espèces de termites du genre Macrotermes ( $M$. subbyalinus séché et $M$. bellicosus frais). Les quantités de matières sèches (MS) et de protéines brutes (PB) fournies par nos différentes rations ont varié de 56,60 à 69,26 et de 9,83 à 11,81 g/jour/individu respectivement. Ces valeurs de MS sont inférieures à celle obtenue par Kingori et al. (2007) tandis que les quantités de PB sont supérieures à la leur. En effet, ces auteurs ont estimé qu'un poulet en croissance pouvait ingérer 78,3 g de MS et 8,5 g de protéines brutes par jour au cours de la divagation. Pour ces mêmes auteurs, les besoins en protéines brutes de la poule locale dans un système d'élevage en liberté s'élèveraient à 11,7 $\mathrm{g} /$ jour/individu soit un apport de 3,2 $\mathrm{g}$ de protéines brutes supplémentaires nécessaires pour une croissance optimale durant 14 à 25 semaines d'âge. Dans une étude antérieure, Kingori et al. (2003) avaient rapporté un besoin en $\mathrm{PB}$ de $10,9 \mathrm{~g}$ dans un système d'élevage en confinement. Nos valeurs de PB sont de cet ordre de grandeur, preuve que nos différentes rations sont convenables pour la période de ponte aussi bien pour l'élevage en claustration qu'en liberté des poules en général.

5.3 Étude de la répartition spatiotemporelle des oiseaux en fonction des préférences alimentaires: Le résultat des observations avec vidéo montre que, les quatre premières semaines, les poulets étaient attirés en premier lieu par les mangeoires renfermant le maïs concassé pour, plus tard, marquer une préférence pour les asticots séchés. Cette attraction vis-à-vis du maïs concassé durant les premières semaines aurait dû induire un taux d'ingestion plus élevé pour le maïs, ce qui n'a pas été le cas. En réalité, grâce à leur bec, les poulets peuvent toucher un aliment afin d'apprécier uniquement ses caractéristiques physiques sans l'ingérer (Picard et al, 2000). Par contre, leur présence autour des asticots séchés du traitement de $08 \mathrm{~h}$ a été suivie d'une augmentation de son ingestion à partir de la 5è semaine. Ce constat peut s'expliquer par une sensibilisation graduelle des poulets au contact des asticots qui, au fil du temps, ont appris à les apprécier (Bouvarel et al., 2010).

5.4 Évaluation des caractéristiques des œufs : La coloration du jaune d'œuf est directement liée à l'alimentation de la poule à cause des pigmentations caroténoïques absorbées par l'animal principalement du maïs jaune (Liu et al., 2012). L'indice de coloration obtenu dans cette étude a été inférieur à celui relevé par Ewonetu et Negassi (2016). Ces auteurs ont rapporté un indice moyen de 8,58 pour les œufs des poulets traditionnels en Éthiopie, un écart pouvant être lié à la différence de concentration en caroténoïdes des maïs utilisés dans les deux études mais aussi à la présence de verdure dans l'environnement. Dans la présente étude, le poids moyen des 
œufs est similaire aux poids des œufs $(41,91 \mathrm{~g}$ et $42,30 \mathrm{~g})$ relevés respectivement par Akouango et al. (2010) au Congo et Ko-Awono et al. (2013) au Cameroun chez des poules traditionnelles. Bien que les valeurs de poids obtenues dans notre cas soient meilleures comparativement à celles $(32,5 \mathrm{~g}$ et $35 \mathrm{~g})$ de Traoré (2006) relevées chez des poulets traditionnels dans des zones péri-urbaines de la ville de Bobo-Dioulasso, elles sont légèrement inférieures à celles $(43,2 \mathrm{~g}$ à 44,0 g) rapportés par Fotsa et al. (2010) au Cameroun en fonction des provinces concernées. Notons que le poids des œufs dépend de l'âge de la poule, de son alimentation et de son poids vif. Le poids du jaune d'œuf obtenu au cours de la présente étude a été supérieur à ceux de Ewonetu et Negassi (2016) ainsi que de Garba et al. (2016) qui ont rapporté respectivement des valeurs de $14,67 \mathrm{~g}$ et $14,07 \mathrm{~g}$ chez des poulets traditionnels en Éthiopie et au Nigeria. Le poids moyen du blanc d'œuf de notre étude a été supérieur à celui de Garba et al. (2016) mais inferieurs aux résultats trouvés par Ewonetu et Negassi (2016). Ces auteurs ont relevé respectivement chez des poulets traditionnels des poids de 19,48 g et 30,27 g. Par contre, le poids moyen des coquilles de cette étude était similaire au 5,51 g trouvé par Ewonetu et Negassi (2016).

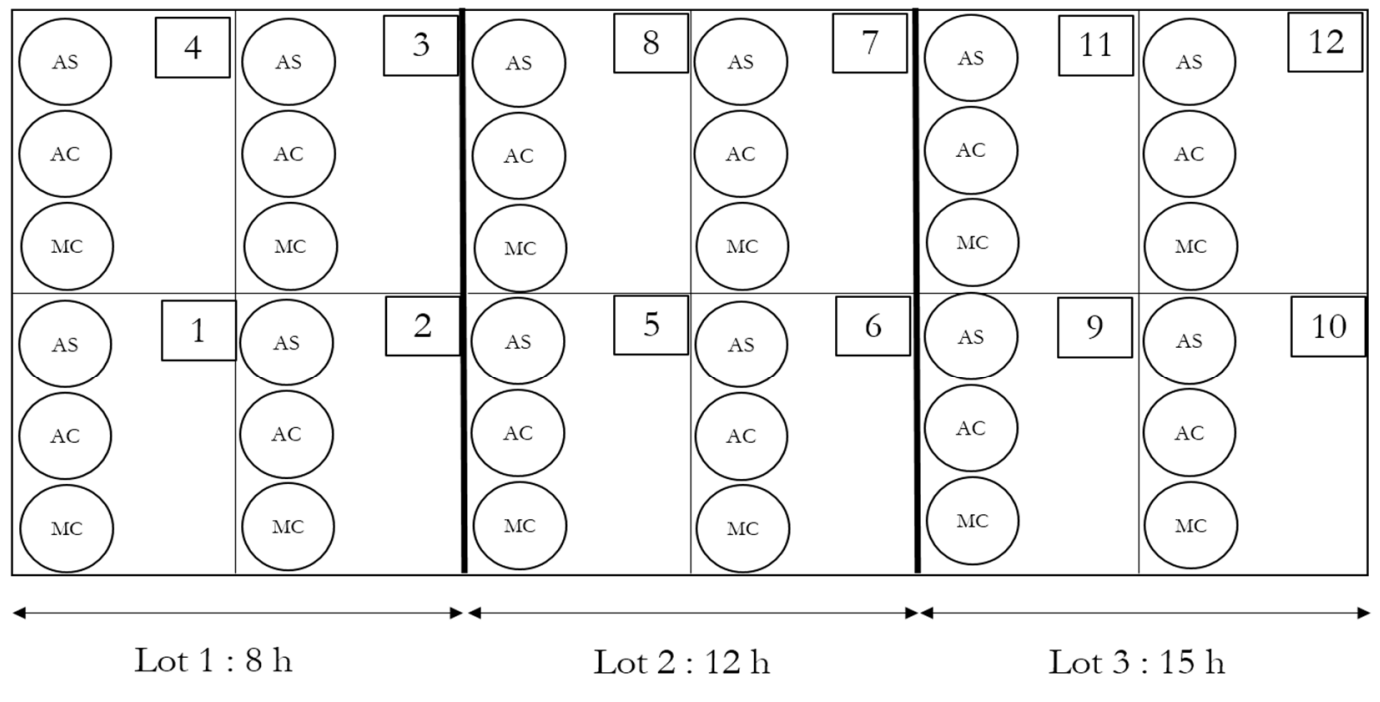

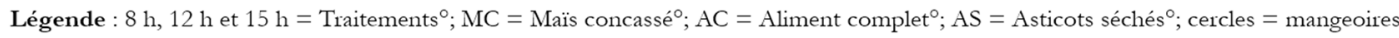

Figure 1 : Dispositif expérimental. 

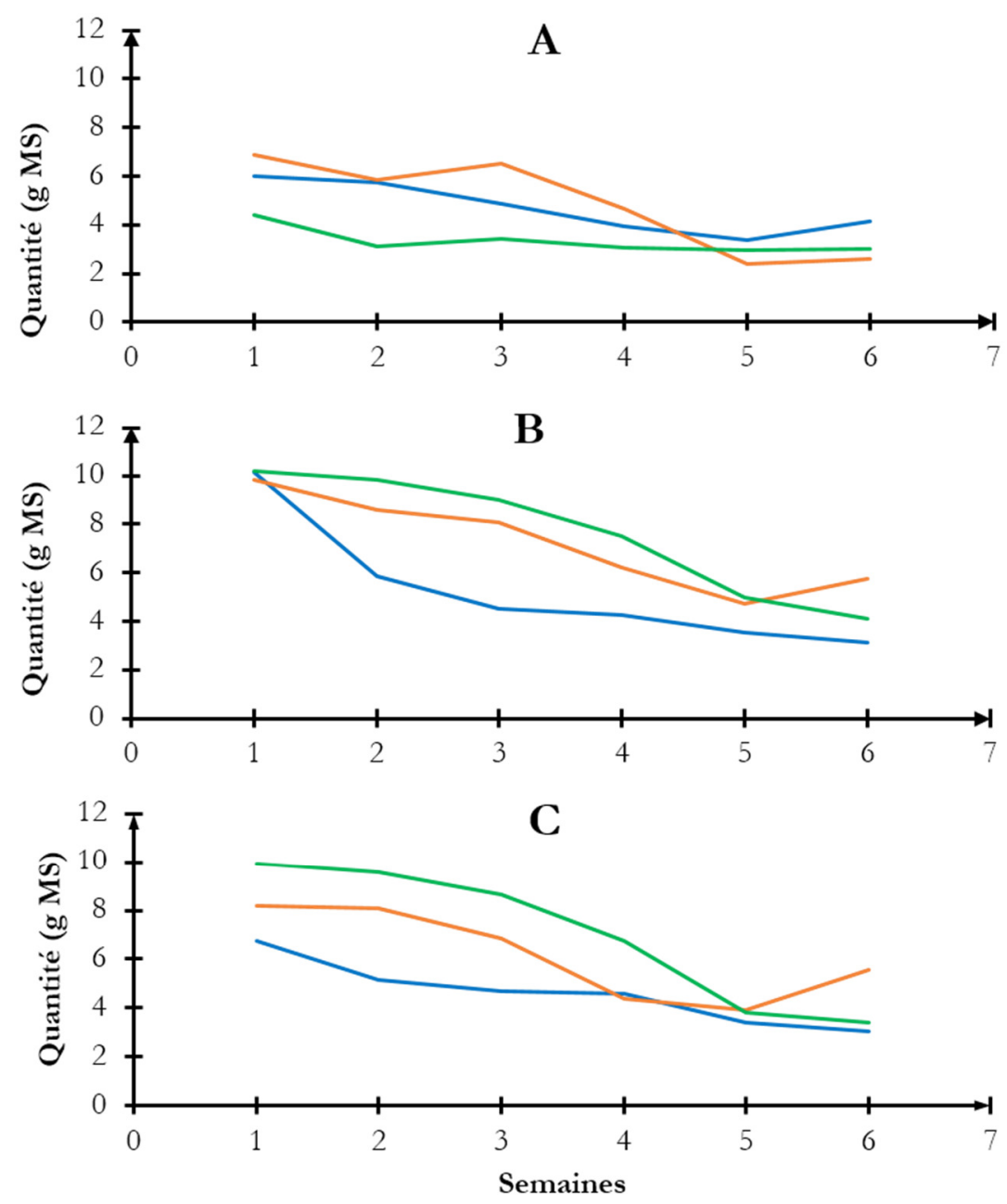

—Asticots séchés Maïs concassé _-Aliment complet

Figure 2 : Évolution des quantités d'aliment consommé en fonction des périodes de service ou traitements $(\mathrm{A}=08 \mathrm{~h}, \mathrm{~B}=12 \mathrm{~h}$ et $\mathrm{C}=15 \mathrm{~h})$.

Tableau 1 : Composition centésimale de l'aliment complet ponte et sa valeur nutritionnelle.

\begin{tabular}{l|c}
\hline Ingrédients & Proportion (\%) \\
\hline Maïs & 61,03 \\
Tourteau de coton & 6,00 \\
Son de blé & 15,00 \\
Farine de poisson & 9,00 \\
Lysine & 0,10 \\
Méthionine & 0,10 \\
Coquille d'huître & 8,00 \\
Sel iodé & 0,21 \\
Phosphate & 0,16 \\
Prémix ponte & 0,30 \\
Sulfate de fer & 0,10 \\
\hline
\end{tabular}


EM (kcal)

PB (\%)

$\mathrm{Ca}(\%)$

2685

16,14

$\mathrm{P}(\%)$

3,79

0,75

Lysine (\%)

Méthionine (\%)

0,86

0,38

$\mathbf{E M}=$ Énergie Métabolisable $; \mathbf{P B}=$ Protéine Brute $; \mathbf{C a}=$ calcium $; \mathbf{P}=$ Phosphate.

Tableau 2 : Variation de la composition chimique des aliments utilisés.

\begin{tabular}{|c|c|c|c|c|c|c|c|c|c|}
\hline Rations & $\% \mathrm{MS}$ & $\% \mathrm{MM}$ & $\% \mathrm{MO}$ & $\% \mathrm{MG}$ & $\%$ PB & $\% \mathrm{CB}$ & $\begin{array}{c}\text { EM } \\
(\mathrm{Kcal} / \mathrm{kg})\end{array}$ & $\begin{array}{c}P \\
(\mathrm{mg} / \mathrm{Kg})\end{array}$ & $\begin{array}{c}\mathrm{Ca} \\
(\mathrm{mg} / \mathrm{Kg})\end{array}$ \\
\hline Asticots & 91,87 & 21,10 & 78,90 & 3,93 & 48,85 & 9,16 & 2491,42 & 2,18 & 5,96 \\
\hline $\begin{array}{l}\text { Maïs } \\
\text { concassé }\end{array}$ & 88,89 & 1,97 & 98,03 & 0,79 & 12,24 & 3,46 & 3606,70 & 862 & 448 \\
\hline ACP & 90,30 & 13,27 & 86,73 & 1,36 & 14,75 & 10,87 & 2519,40 & 1601 & 10080 \\
\hline
\end{tabular}

$\mathrm{ACP}=$ Aliment complet ponte ; MS : matière sèche ; MM : matière minérale ; $\mathrm{MO}$ : matière organique ; MG : matière grasse; $\mathrm{PB}$ : protéine brute; $\mathrm{CB}$ : cellulose brute; $\mathrm{EM}$ : énergie métabolisable calculée; $\mathrm{P}=$ Phosphore $; \mathrm{Ca}=$ Calcium.

Tableau 3: Variation des quantités d'aliments consommés (g MS/jr/individu) en fonction des périodes de service.

\begin{tabular}{c|c|c|c}
\hline Traitements & Asticots séchés & Maïs concassé & Aliment complet \\
\hline $08 \mathrm{~h}$ & $4,68 \pm 1,81 \mathrm{~b}$ & $4,83 \pm 2,54 \mathrm{c}$ & $3,32 \pm 1,13 \mathrm{~b}$ \\
$12 \mathrm{~h}$ & $5,23 \pm 2,59 \mathrm{a}$ & $7,19 \pm 2,24 \mathrm{a}$ & $7,59 \pm 2,75 \mathrm{a}$ \\
$15 \mathrm{~h}$ & $4,60 \pm 1,67 \mathrm{~b}$ & $6,16 \pm 2,21 \mathrm{~b}$ & $7,02 \pm 2,97 \mathrm{a}$ \\
\hline Moyenne générale & $4,84 \pm 2,08$ & $6,06 \pm 2,53$ & $5,97 \pm 3,07$ \\
\hline \multirow{2}{*}{ Statistiques } & $\mathrm{F}=4,686$ & $\mathrm{~F}=43,090$ & $\mathrm{~F}=153,200$ \\
& $\mathrm{P}=0,0096$ & $\mathrm{P}<2 \mathrm{e}-16$ & $\mathrm{P}<2 \mathrm{e}-16$ \\
& $\mathrm{Ddl}=2$ & $\mathrm{Ddl}=2$ & $\mathrm{Ddl}=2$ \\
\hline
\end{tabular}

Dans la même colonne, les moyennes suivies de la même lettre ne sont pas significativement différentes au seuil de probabilité de 5\% selon le test de Student.

Tableau 4 : Variation du taux (\%) des différents aliments consommés selon le traitement.

\begin{tabular}{c|c|c|c}
\hline Traitements & Asticots séchés & Maïs concassé & Aliment complet \\
\hline $08 \mathrm{~h}$ & $36,48 \mathrm{a}$ & $37,65 \mathrm{a}$ & $25,88 \mathrm{c}$ \\
$12 \mathrm{~h}$ & $26,14 \mathrm{~b}$ & $35,93 \mathrm{~b}$ & $37,93 \mathrm{~b}$ \\
$15 \mathrm{~h}$ & $25,89 \mathrm{~b}$ & $34,67 \mathrm{c}$ & $39,50 \mathrm{a}$ \\
\hline Taux moyen $(\%)$ & 28,69 & 35,92 & 35,39 \\
\hline
\end{tabular}

Dans la même colonne, les moyennes suivies de la même lettre ne sont pas significativement différentes au seuil de probabilité de 5\% selon le test de Khi2. 
Tableau 5: Variation de la consommation alimentaire journalière totale, des quantités d'aliments consommés pendant et après la cafétéria (en g MS/individu) et leurs taux (\%) selon les traitements.

\begin{tabular}{|c|c|c|c|c|}
\hline \multicolumn{2}{|c|}{ Traitements } & $\begin{array}{l}\text { Consommation } \\
\text { de la cafeteria }\end{array}$ & $\begin{array}{l}\text { Aliment complet } \\
\text { après le test }\end{array}$ & $\begin{array}{c}\text { Consommation alimentaire } \\
\text { journalière totale }\end{array}$ \\
\hline $08 \mathrm{~h}$ & $\begin{array}{l}\text { Moyenne } \\
\text { Taux }(\%)\end{array}$ & $\begin{array}{c}12,83 \pm 4,35 c \\
22,67\end{array}$ & $\begin{array}{c}43,77 \pm 9,45 b \\
77,33\end{array}$ & $\begin{array}{c}56,60 \pm 12,08 b \\
100\end{array}$ \\
\hline $12 \mathrm{~h}$ & $\begin{array}{l}\text { Moyenne } \\
\text { Taux }(\%)\end{array}$ & $\begin{array}{c}20,01 \pm 6,75 a \\
28,89\end{array}$ & $\begin{array}{c}49,25 \pm 10,68 \mathrm{a} \\
71,11 \\
\end{array}$ & $\begin{array}{c}69,26 \pm 16,53 a \\
100\end{array}$ \\
\hline $15 \mathrm{~h}$ & $\begin{array}{l}\text { Moyenne } \\
\text { Taux }(\%)\end{array}$ & $\begin{array}{c}17,78 \pm 5,98 b \\
26,97\end{array}$ & $\begin{array}{c}48,14 \pm 10,08 \mathrm{a} \\
73,03\end{array}$ & $\begin{array}{c}65,92 \pm 15,07 a \\
100\end{array}$ \\
\hline \multicolumn{2}{|c|}{$\begin{array}{c}\text { Moyenne générale } \\
\text { Taux }(\%)\end{array}$} & $\begin{array}{c}16,87 \pm 6,50 \\
26,39\end{array}$ & $\begin{array}{c}47,05 \pm 10,34 \\
73,60\end{array}$ & $\begin{array}{c}63,93 \pm 15,60 \\
100\end{array}$ \\
\hline \multicolumn{2}{|c|}{ Statistiques } & $\begin{array}{l}F=67,90 \\
P<2 e-16 \\
D d l=2\end{array}$ & $\begin{array}{l}F=13,88 \\
P=1,36 \mathrm{e}-16 \\
D d l=2\end{array}$ & $\begin{array}{l}\mathrm{F}=33,59 \\
\mathrm{P}=2,04 \mathrm{e}-14 \\
\mathrm{Ddl}=2\end{array}$ \\
\hline
\end{tabular}

Dans la même colonne, les moyennes suivies de la même lettre ne sont pas significativement différentes au seuil de probabilité de 5\% selon le test de Student.

Tableau 6: Variation des quantités de nutriments dans les rations selon les traitements et par individu.

\begin{tabular}{|c|c|c|c|c|c|c|c|c|c|c|}
\hline \multirow{2}{*}{\multicolumn{2}{|c|}{ Traitements }} & \multicolumn{9}{|c|}{ Nutriments } \\
\hline & & \multirow{2}{*}{$\begin{array}{c}\begin{array}{c}\text { MS } \\
(\mathbf{g})\end{array} \\
12,83\end{array}$} & \multirow{2}{*}{$\begin{array}{c}\begin{array}{c}\text { MM } \\
(\mathbf{g})\end{array} \\
1,52\end{array}$} & \multirow{2}{*}{$\begin{array}{c}\text { MO } \\
\text { (g) } \\
11,31\end{array}$} & \multirow{2}{*}{$\begin{array}{r}\begin{array}{r}\text { MG } \\
(\mathbf{g})\end{array} \\
0,27\end{array}$} & \multirow{2}{*}{$\begin{array}{c}\text { PB } \\
\text { (g) } \\
3,37\end{array}$} & \multirow{2}{*}{$\begin{array}{l}\text { CB } \\
\text { (g) }\end{array}$} & \multirow{2}{*}{$\begin{array}{c}\begin{array}{c}\text { EM } \\
\text { (Kcal/kg MS) }\end{array} \\
3818,52\end{array}$} & \multirow{2}{*}{$\begin{array}{c}\begin{array}{c}\mathbf{P} \\
(\mathbf{m g})\end{array} \\
9,49\end{array}$} & \multirow{2}{*}{$\begin{array}{c}\begin{array}{c}\mathrm{Ca} \\
(\mathbf{m g})\end{array} \\
35,66\end{array}$} \\
\hline \multirow{3}{*}{$08 \mathrm{~h}$} & ATC & & & & & & & & & \\
\hline & ACAT & 43,77 & 5,81 & 37,96 & 0,60 & 6,46 & 4,76 & 3324,38 & 70,08 & 441,20 \\
\hline & CJT & 56,60 & 7,33 & 49,27 & 0,87 & 9,83 & 5,72 & 3191,90 & 79,57 & 476,86 \\
\hline \multirow{3}{*}{$12 \mathrm{~h}$} & ATC & 20,01 & 2,25 & 17,76 & 0,37 & 4,55 & 1,55 & 3741,84 & 18,36 & 79,76 \\
\hline & ACAT & 49,25 & 6,54 & 42,71 & 0,67 & 7,26 & 5,35 & 3246,07 & 78,85 & 496,44 \\
\hline & CJT & 69,26 & 8,79 & 60,47 & 1,04 & 11,81 & 6,90 & 3036,91 & 97,21 & 576,20 \\
\hline \multirow{3}{*}{$16 \mathrm{~h}$} & ATC & 17,78 & 2,02 & 15,76 & 0,32 & 4,04 & 1,40 & 3761,81 & 16,56 & 73,55 \\
\hline & ACAT & 48,14 & 6,39 & 41,75 & 0,65 & 7,10 & 5,23 & 3261,75 & 77,07 & 485,25 \\
\hline & CJT & 65,92 & 8,41 & 57,50 & 0,97 & 11,14 & 6,63 & 3072,56 & 93,63 & 558,80 \\
\hline
\end{tabular}

ATC $=$ Aliments tests de la cafétéria $;$ ACAT $=$ Aliment complet après le test $;$ CJT $=$ Consommation journalière totale $; \mathrm{MS}=$ Matière sèche $; \mathrm{MM}=$ Matière minérale $; \mathrm{MO}=$ Matière organique $; \mathrm{MG}=$ Matière grasse $; \mathrm{PB}=$ Protéines brutes $; \mathrm{CB}=$ Cellulose brute $; \mathrm{EM}=$ Énergie métabolisable $; \mathrm{P}=$ Phosphore $; \mathrm{Ca}=$ Calcium.

Tableau 7 : Effet des traitements sur la caractéristique des œufs (moyenne \pm écart-type).

\begin{tabular}{l|c|c|c|c|c}
\hline Paramètres & $\mathbf{0 8 ~ h}$ & $\mathbf{1 2} \mathbf{h}$ & $\mathbf{1 5} \mathbf{~}$ & $\mathbf{G l o b a l e}$ & $\mathbf{N}$ \\
\hline Poids des œufs (g) & $41,00 \pm 4,18$ & $43,44 \pm 2,99$ & $41,75 \pm 3,51$ & $42,06 \pm 3,66$ & 48 \\
Poids du blanc (g) & $19,94 \pm 3,64$ & $21,50 \pm 3,65$ & $21,69 \pm 3,94$ & $21,04 \pm 3,75$ & 48 \\
Poids du jaune (g) & $15,25 \pm 2,24$ & $16,19 \pm 2,01$ & $14,63 \pm 2,09$ & $15,35 \pm 2,17$ & 48 \\
Poids de la coquille (g) & $5,81 \pm 0,66$ & $5,75 \pm 0,45$ & $5,44 \pm 0,51$ & $5,67 \pm 0,56$ & 48 \\
Coloration du jaune & $7,94 \pm 2,35$ & $8,31 \pm 1,96$ & $7,00 \pm 1,32$ & $7,75 \pm 1,96$ & 48 \\
\hline
\end{tabular}


Sur la même ligne, les moyennes des paramètres ne sont pas significativement différentes au seuil de probabilité de 5\% selon le test de Student.

\section{CONCLUSION}

La présente étude est une contribution pour l'utilisation des asticots de mouches dans l'alimentation des poulets traditionnels. Ces asticots constituent une source de protéines, moins chère et très accessible. Au terme de cette étude, il ressort que les poulets locaux, contrairement aux pintades, apprécient les larves de mouches séchées et l'observation de leur comportement alimentaire montre que ceux-ci apprennent à apprécier les asticots séchés avec le temps. Bien que les écarts de

\section{REMERCIEMENTS}

Cette étude a été réalisée avec l'appui du projet IFWA (Sustainable use of insects to improve livestock production and food security in smallholder farms in West Africa) financé par la Direction du développement et de la coopération Suisse et le Fonds national Suisse de la recherche scientifique, dans le cadre de "Swiss Programm for Research on Global Issues for Development (R4D)". Les auteurs

\section{REFERENCES}

Akouango F, Bandtaba P. et Ngokaka C : 2010. Croissance pondérale et productivité de la poule locale Gallus domesticus en élevage fermier au Congo. Animal Genetic Resources Information 46: 61-65. DOI: $10.1017 / \mathrm{S} 2078633610000706$.

AOAC (Association of official analytical chemists): 1995. Official Methods of Analysis of the Association of Official Analytical Chemistry (16th Edn). AOAC International: Washington.

Bloukounon-Goubalan AY, Saïdou A, Obognon N, Amadji GL, Igué AM, Clottey VA, Chrysostome CAAM, Kenis M. and Mensah GA: 2019. Decomposition and nutrient release pattern of agro-processing by-products biodegraded by fly larvae in Acrisols. Archives of Agronomy and Soil Science 65 consommations soient faibles, cette étude a pu montrer à travers les ingestions que la période de la journée la mieux indiquée pour faire une complémentation en asticots séchés se situe autour de $12 \mathrm{~h}$. Pour une utilisation efficiente des asticots par les animaux et les producteurs, l'évaluation des performances pondérales et de ponte de la volaille nourrie à base d'asticots permettra de mieux vulgariser la technique de production en masse des asticots en milieu paysan.

remercient en particulier le professeur G. A. Mensah pour ses précieux commentaires et sa contribution à la réalisation de cette étude. Marc Kenis a été partiellement financé par le CABI Development Fund (contributions du "Australian Centre for International Agricultural Research", "UK's Department for International Development", et autres).

(11): $\quad$ 1610-1621. DOI: https://doi.org/10.1080/03650340.201 $\underline{9.1572118}$.

Bouvarel I, Tesseraud S. et Leterrier C : 2010. L'ingestion chez le poulet de chair: n'oublions pas les régulations à court terme. INRA Prod. Anim. 23 (5) : 391404.

https://prodinra.inra.fr/record/46505.

Bouvarel I, Lessire M, Narcy A, Duval E, Grasteau S, Quinsac A, Peyronnet C, Tran G. et Heuze V : 2014. Des sources de protéines locales pour l'alimentation des volailles : quelles voies de progrès ?, Oilseeds \& fats Crops and Lipids 21 (4) D405.

DOI: https://doi.org/10.1051/ocl/2014014.

Dao ANC, Sankara F, Pousga S, Coulibaly K, Nacoulma JP, Ouédraogo S., Kenis M. 
and Somda I: 2020. Traditional methods of harvesting termites used as poultry feed in Burkina Faso. Int. J. Trop. Insect Sci. Available online. DOI: https://doi.org/10.1007/s42690-01900059-w.

Ewonetu KS. and Negassi AZ: 2016. Evaluation of external and internal quality of chicken table eggs at retailers in eastern Ethiopia. J. Anim. Sci. Adv. 6 (5): $\quad$ 1642-1649. DOI: $10.5455 /$ jasa.20160524124303.

Fanatico AC, Brewer VB, Owens-Hanning CM, Donoghue DJ. and Donoghue AM: 2013. Free-choice feeding of free-range meat chickens. J. Appl. Poult. Res. 22:750-758.

DOI:

http://dx.doi.org/10.3382/japr.2012$\underline{00687}$.

Fanatico AC, Owens-Hanning CM, Brewer Gunsaulis V. and Donoghue AM: 2016. Choice feeding of protein concentrate and grain to organic meat chickens. $J$. Appl. Poult. Res. 25: 156-164. DOI : http://dx.doi.org/10.3382/japr/pfv076

Fasakin EA, Balogun AM. and Ajayi OO: 2003. Evaluation of full-fat and defatted maggot meals in the feeding of clariid cat fish Clarias gariepinus fingerlings. Aquaculture and Research 34:733-738. DOI: $\quad 10.1046 / \mathrm{j} .1365$ 2109.2003.00876.x.

Fotsa JC, Rognon X, Tixier-Boichard M, Coquerelle G, Poné Kamdem D, Ngou Ngoupayou JD, Manjeli Y. et Bordas A : 2010. Caractérisation phénotypique des populations de poules locales (Gallus) de la zone forestière dense humide à pluviométrie bimodale du Cameroun. Animal Genetic Resources Information 46: 49-59. DOI: http://dx.doi.org/10.1017/S207863361 000069X.

Garba S, Assam ED, Yusuf RO, Sa'adu A. and Jibir M: 2016. Compararative evaluation of eggs from three selected species of indigenous laying hens. Livestock Research for Rural Development 28 (5). http://www.lrrd.org/lrrd28/5/gard280 76.htm.

Guinko S: 1984. Végétation de la Haute-Volta. Thèse de Doctorat en Sciences Naturelles, Université de Bordeaux II, France, 394 pp.

INRA (Institut National de la Recherche Agronomique) de France: 1987. Nutrition of laying hens. In: J. Wiseman (ed) Feeding of Non-Ruminant Livestock. Butterworths and Co Ltd: London.

INSD (Institut National de la Statistique et de la Démographie): 2018. Annuaire statistique 2017 de la région des Hauts Bassins. INSD, Ministère de l'économie, des finances et du développement (MINEFID), Burkina Faso, 269 pp.

Kenis M, Koné N, Chrysostome CAAM, Devic E, Koko GKD, Clottey VA, Nacambo S. and Mensah GA: 2014. Insects used for animal feed in West Africa. Entomologia 2 (218): 107-114. DOI: 10.4081/entomologia.2014.218.

Kenis M, Bouwassi B, Boafo H, Devic E, Han R, Koko G, Koné N'G, Maciel-Vergara G, Nacambo S, Pomalegni SCB, Roffeis M, Wakefield M, Zhu F. and Fitches E: 2018. Small-scale fly larvae production for animal feed. In: Halloran A, Flore R, Vantomme P. and Roos N (Eds.) Edible insects in Sustainable Food Systems. Springer 239-261. DOI: https://doi.org/10.1007/978-3-31974011-9 15 .

Kingori AM, Tuitoek JK, Muiruri HK. and Wachira AM: 2003. Protein requirements of growing indigenous chickens during the 14-21 week growing period. South African J. Anim. Sci. 33 (2): 78-82. DOI: $10.4314 /$ sajas.v33i2.3759.

Kingori AM, Tuitoek JK, Muiruri HK, Wachira AM. and Birech EK: 2007. Protein intake of growing indigenous chickens on free-range and their response to 
supplementation. Int. J. Poult. Sci. 6 (9): 617-621. DOI: 10.3923/ijps.2007.617.621.

Ko-Awono PMD, Fotsa JC, Njehoya Aggy C, Awa Ndzingu D, Njoya A. and Ngo Tama AC: 2013. Caractérisation phénotypique des populations traditionnelles de poules dans la zone soudano-sahélienne du Cameroun. Family Poult. Commun. 22 (1): 11-17.

Liu Y-Q, Davis CR, Schmaelzle ST, Rocheford T, Cook ME. and Tanumihardjo SA: 2012. $\beta$-Cryptoxanthin biofortified maize (Zea mays) increases $\beta$ cryptoxanthin concentration and enhances the color of chicken egg yolk. Poult. Sci. 91 (2): 432-438. DOI: https://doi.org/10.3382/ps.2011$\underline{01719}$.

Makkar HPS, Tran G, Heuzé V. and Ankers P: 2014. State-of-the-art on use of insects as animal feed. Anim. Feed Sci. Tech. 197: $1-33$.

DOI : http://dx.doi.org/10.1016/j.anifeedsci. 2014.07.008.

MRA (Ministère des Ressources Animales): 2015. Annuaire des statistiques de l'élevage 2013-2014. Direction des statistiques sectorielles, Ouagadougou, Burkina Faso. 177 pp.

Ouattara S, Bougouma-Yaméogo VMC, Nianogo AJ. and Savadogo B: 2016. Influence of substituting roasted soybean (Glycine max) seeds by those of cowpea (Vigna unguiculata) and of the protein level in the diet on the performance of the local-breed guinea fowl in Burkina Faso. Rev. Elev. Med. Vet. Pays Trop. 69 (3) : 117-123. DOI : http://dx.doi.org/10.19182/remvt.3119 $\underline{5}$.

Picard M, Le Fur C, Melcion JP. et Bouchot C : 2000.

Caractéristiques

Granulométriques de l'aliment : le « point de vue » (et de toucher) des volailles. INRA Prod. Anim. 13 (2) : 117 -
130.

https://prodinra.inra.fr/record/66736. Pomalégni SCB, Gbemavo DSJC, Kpadé CP, Babatoundé S, Chrysostome CAAM, Koudandé

Kenis M, Glèlè KRL. et Mensah GA : 2016. Perceptions et facteurs déterminant l'utilisation des asticots dans l'alimentation des poulets locaux (Gallus gallus) au Bénin. J. Appl. Biosci. 98: 9330 9343.

DOI : http://dx.doi.org/10.4314/jab.v98i1.9.

Pousga S, Boly H. et Ogle B: 2005. Choice Feeding of Poultry: A review. Livestock Research for Rural Development 17 (4). http://www.lrrd.org//rrd17/4/pous170 45.htm.

Pousga S, Boly H, Lindberg JE. and Ogle B: 2007. Evaluation of traditional sorghum (Sorghum bicolor) beer residue, shea-nut (Vitellaria paradoxa) cake and cottonseed (Gossypium spp) cake for poultry in Burkina Faso: Availability and aminoacid digestibility. Int. J. Poult. Sci. 6 (9): 666-672.

DOI : http://dx.doi.org/10.3923/ijps.2007.66 $\underline{6.672}$.

Pousga S, Sankara F, Coulibaly K, Nacoulma JP, Ouédraogo S, Kenis M, Chrysostome CAAM. et Ouédraogo GA: 2019. Effets du remplacement de la farine de poisson par les termites (Macrotermes sp.) sur l'évolution pondérale et les caractéristiques de carcasse de la volaille locale au Burkina Faso. Afr. J. Food Agric. Nutr. Dev. 19 (2) : 14354-14371. DOI : http://dx.doi.org/10.18697/ajfand.85.1 7430.

Sankara F, Pousga S, Dao NCA, Gbemavo DSJC, Clottey VA, Coulibaly K, Nacoulma JP, Ouédraogo S. and Kenis $\mathrm{M}$ : 2018. Indigenous knowledge and potential of termites as poultry feed in Burkina Faso. J. Insects Food and Feed 4: 211-218. DOI: 10.3920/JIFF2017.0070. 
Sanou AG, Sankara F, Pousga S, Coulibaly K, Nacoulma JP, Ouédraogo I, Nacro S, Kenis M, Sanon A. et Somda I : 2019a. Production de masse de larves de Musca domestica L. (Diptera : Muscidae) pour l'aviculture au Burkina Faso ${ }^{\circ}$ : Analyse des facteurs déterminants en oviposition naturelle. J. Appl. Biosci. 134: 1368913701.

DOI : https://dx.doi.org/10.4314/jab.v134i1. $\underline{6}$.

Sanou AG, Sankara F, Pousga S, Kenis M, Coulibaly K, Nacoulma JP, Nacro S, Ouédraogo I. and Somda I: 2019b. Farmers' perception of the use of fly larvae in poultry feed in Burkina Faso. African Entomology 27 (2): 373-385. DOI: https://doi.org/10.4001/003.027.0373.

Tendonkeng F, Miégoué E, Lemoufouet J, Mouchili M, Matimuini NF, Mboko AV, Fogang Zogang B, Mweugang NN, Zougou TG, Boukila B. et Pamo TE: 2017. Production et composition chimique des asticots en fonction du type de substrat. Livestock Research for Rural Development, $29 \quad$ (4). http://www.lrrd.org/lrrd29/4/ften290 67.html

Traoré B : 2006. Effet des parasites internes sur la productivité des volailles locales ${ }^{\circ}$ : cas des helminthes. Mémoire du Diplôme d’Ingénieur du Développement Rural. Institut du Développement Rural/Univ. Polytechnique de Bobo-Dioulasso, Burkina Faso, 67pp.

Traoré I, Pousga S, Sankara F, Coulibaly K, Nacoulma JP, Kenis M, Mensah GA. et Ouédraogo $\mathrm{GA}^{\circ}$ : 2020. Étude du comportement alimentaire de la pintade locale (Numida meleagris, L.) à l'Ouest du Burkina Faso. Int. J. Biol. Chem. Sci. 14 (1): 154-169. DOI: https://dx.doi.org/10.4314/ijbcs.v14i1. 13.

van Huis A, Van Itterbeck J, Klunder $H$, Mertens E, Halloran A, Muir G. and Vantomme P: 2013. Edible insects future prospects for food and feed security. FAO, Forestry Paper 171, Rome, Italy, 187pp. 